\title{
Decision support in maintenance activity by using modern techniques for equipment condition monitoring
}

\author{
Ionel Paunescu, Paul Liviu Paunescu, and Ştefan Velicu* \\ University Politehnica of Bucharest, Romania
}

\begin{abstract}
As the complexity of the equipment and embedded technologies grow, the decision to take action for keeping the normal operation condition is increasingly challenging. The development of methods and instruments meant to improve availability, reliability and maintainability of equipment involves research on the of the related subassemblies and components in various conditions of operation. To maximize technical system performance, to ensure the operational safety and to decrease functioning costs, the authors of this paper propose the implementation of some monitoring techniques, using technical indicators specific to the system (markers for maintenance) and an innovative process based on the electricity footprint method for monitoring the operational behavior of the critical items in a kinematic chain. The analysis of the monitoring techniques presented in this paper leads to an effective act for keeping the normal operation condition of a technical system.
\end{abstract}

\section{Introduction}

Starting with the $9^{\text {th }}$ decade of the $20^{\text {th }}$ century, many industrialized countries used the specialized software products for technical systems maintenance. In our country, it took a long time to show that the maintenance of technical systems is included in the category of computer-based activities.

The paper [1] specifies that the management of maintenance works takes into account several approaches, namely:

- proactive scheduling and planning;

- team work;

- contractors-oriented productivity;

- continuous improvement of maintenance process;

- continuous improvement of materials and services purchase process.

* Corresponding author: velstefan@hotmail.com 
To maintain the operational status of a technical system it is necessary to consider the existing types of maintenance, namely: scheduled maintenance and unscheduled maintenance.

These two large types of maintenance are classified into groups and subgroups as follows:

A) Group 1- unscheduled: - reactive.

B) Group 2- scheduled: - preventive,

- proactive

- by monitoring;

- scheduled.

The first group (A) has a short path to follow, but also a major drawback difficult to control during the production process, namely the action taken when a component of the technical system fails. Although the costs of maintenance operations in this variant are reduced to labor costs and to the equivalent value of the defective component or group of components, the overall costs for the re-commissioning of the technical system are increased with the costs of machine down-time and also the costs of the damages caused by stopping the manufacturing line (when there is no backup equipment) [1] [4].

The second group (B) has a more complex path to follow, the costs for maintenance operations are higher because of monitoring equipment and staff specialization, but the moment of intervention in the equipment can be controlled [1].

Regarding these techniques that currently use modern but expensive instrumentation (Figure 1), this research paper puts particular emphasis on the implementation of sensors on certain critical components of the technical system, the monitoring of preset parameters by means of cheap and user-friendly instrumentation that reduces the operational risks [2].

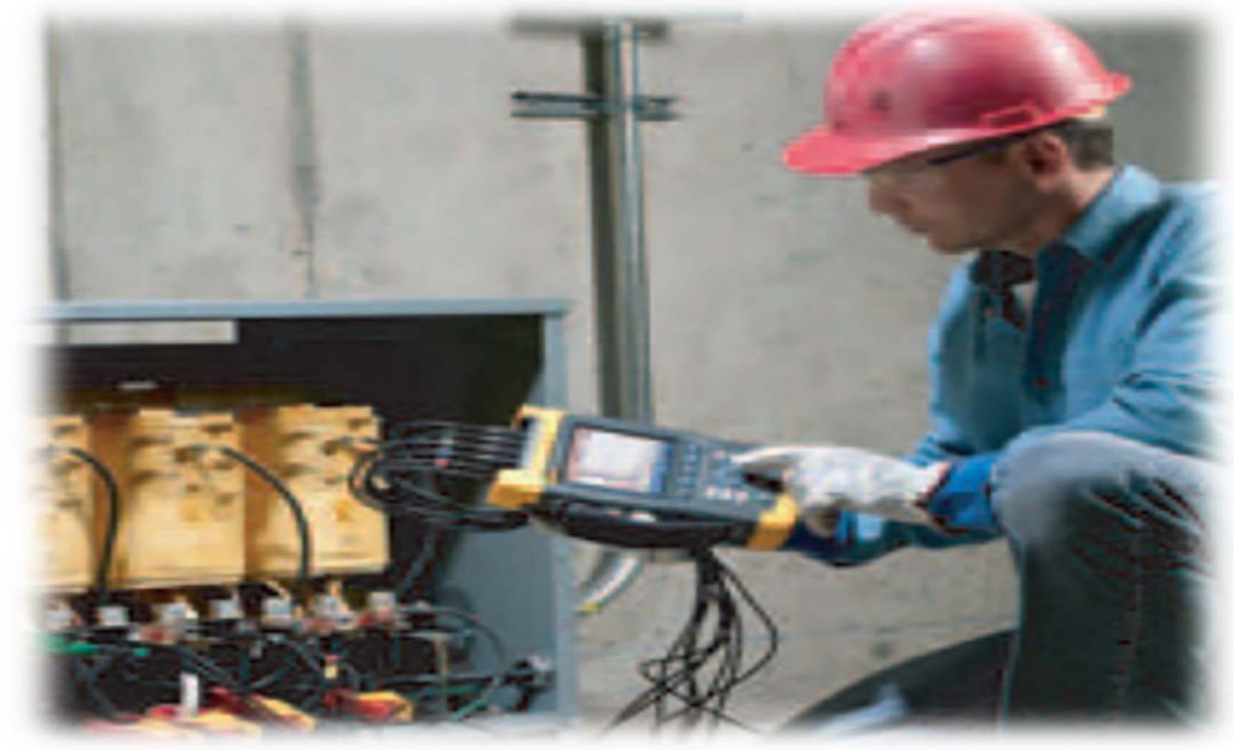

Fig. 1. Monitoring instrumentation (Fluke 430 Series, power quality analyzer).

For maintaining the equipment operating costs at the value of proper operation period it is effective to use the method of monitoring some technical indicators specific to the system (maintenance markers) which lead us to balance the result obtained with the sustainable costs (Figure 2). 


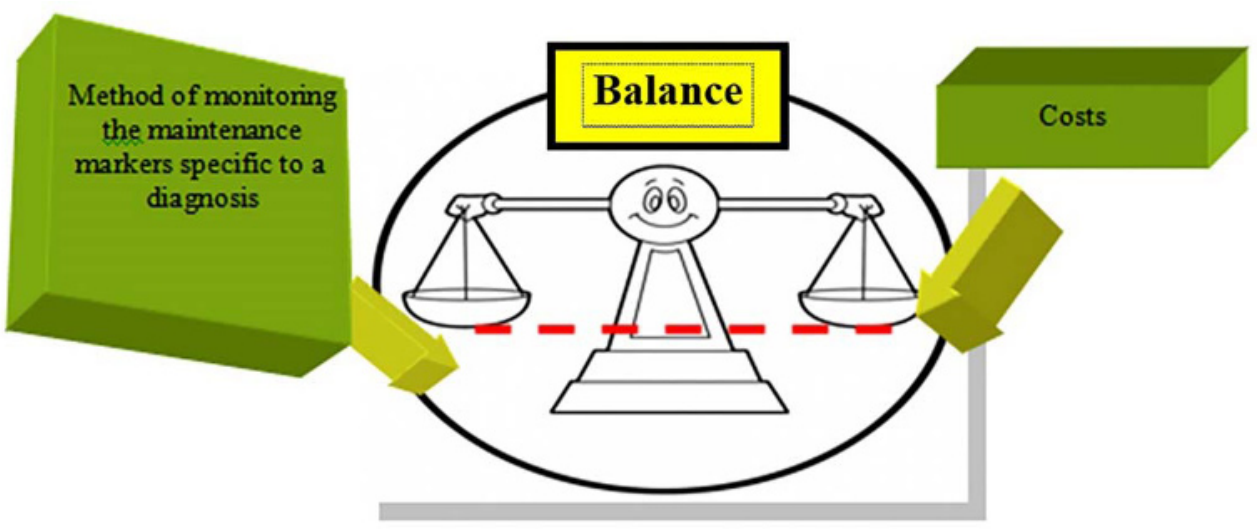

Fig. 2. Balance of maintenance.

\section{Monitoring system of kinematic chain faults based on electricity footprint method as maintenance marker}

To simulate the operation of a kinematic chain it has been developed the dynamic model of this one (Figure 3). This model basically describes the relationship between the instantaneous angular position of each shaft and the torque of the electric motor reflected in the motor shaft and electric parameters (voltage, current). Any disturbance of the rotational movement of the kinematic chain is transmitted to the motor shaft. By monitoring the current absorbed by the motor and the rotational speed of a shaft it is possible to determine accurately which is the mechanism that generates disturbances (gearing, bearings, misalignments etc.) and the evolution of degradation over time.

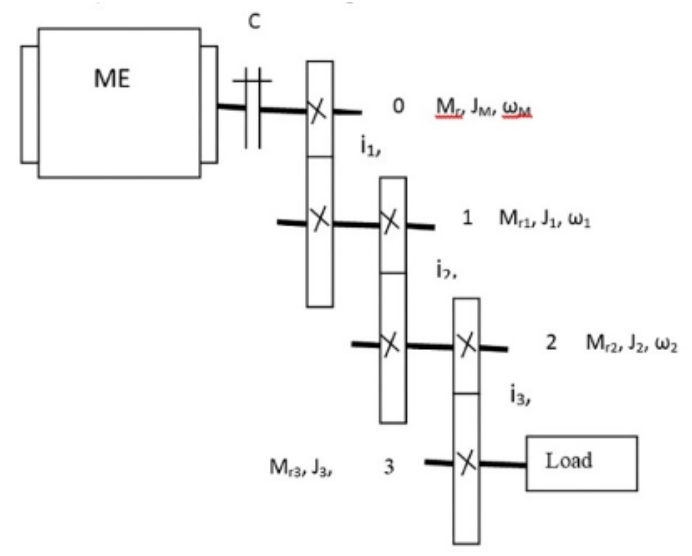

Fig. 3. Dynamic model of the kinematic chain.

Let us assume that a certain constraint to a rotation of the main shaft, starting by the angle $\theta_{3}$, results in the variation of the speed, namely of the angular velocity $\omega$ over a time $\Delta t$.

This variation of the angular velocity determines an angular acceleration $\varepsilon_{3}$, where:

$$
\varepsilon_{3}=\frac{d \omega_{3}}{d t}
$$


which shall be propagated in the motor shaft:

$$
\varepsilon_{M}=\frac{d \omega_{3}}{d t} \cdot i_{1} \cdot i_{2} \cdot i_{3}
$$

In this case, the dynamic torque in motor shaft will be:

$$
M_{\text {din }}=J \cdot \varepsilon_{M}
$$

The angular velocity can be expressed depending on speed:

$$
\omega=\frac{2 \cdot \pi \cdot n}{60}
$$

In which case the dynamic torque will be:

$$
M_{\text {din }}=J \cdot \frac{d n_{M}}{30}
$$

This dynamic torque will entail a variation of the motor shaft power:

$$
\Delta P_{\text {din }}=M_{\text {din }} \cdot \Delta \omega_{M}
$$

Given the constant voltage (Uconst), the power variation will be seized by the current variation $\Delta \mathrm{I}$. The change of current intensity size measured at the main electric motor is a consequence of changes in the operational parameters of some elements that compose the technical system. In this case, the aforementioned equations are changed accordingly. Going on reverse logic we can reveal the components that disturb the rotational movement (bearings, gearing, wear, eccentric masses etc.) and thus a maintenance decision can be taken in order to keep the normal operating parameters of the equipment [5].

\section{Experimental researches}

Using these data, the authors propose a method to measure the parameters of the electric motor for kinematic chain drive (current, rotational speed and temperature) and to determine a relationship between the variation of these parameters and the proper operation of movement transmission mechanisms. The hypothesis was verified on an experimental stand so designed to be able to simulate different conditions of failure of kinematic chain components: uniform and nonuniform wear of a bearing, coaxiality error of motor coupling, an eccentric mass on a shaft, gearing error or combinations of these ones. The system includes a module for data acquisition, represented by an interface NI-DAQ 6251 (b), connected to a computer(a) via USB port, an extension module(c) which contains sensors for measuring the current, voltage and temperature of the motor, and also a LabVIEW software application for processing and representing the information recorded by the acquisition module [3].

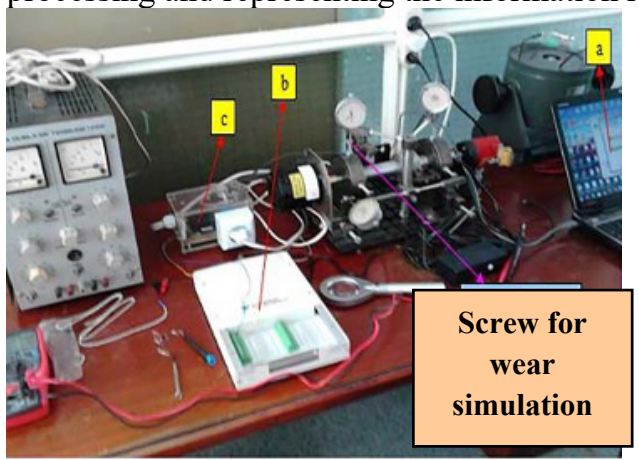

a)

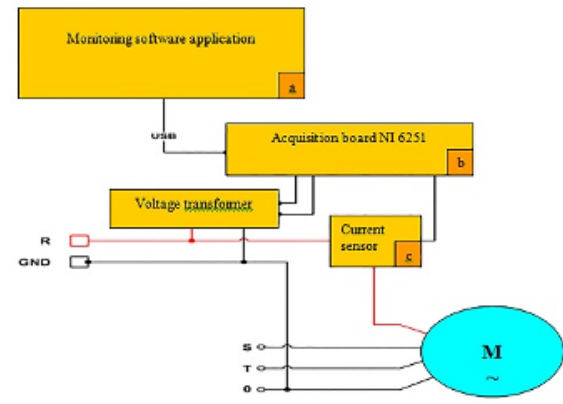

b)

Fig. 4. Monitoring system of electricity footprint ( $a$ - research stand, $b$ - block diagram). 
The research paper focuses on studying the thermal and mechanical behavior of the electric motor, transmitted by the elements of a kinematic chain in normal status and in wear conditions too. The electric signals obtained by using LabVIEW software, adapted to this research paper, were analyzed and compared with the standard signals obtained for "NORMAL" status - MT (machine tool) operation within the initial parameters, according to the Instructions Book [3].

The shape of the signals obtained is structured as follows:

a) constant amplitude of the signal displayed (with determined values, up to the value of $800 \mathrm{~mA}$ ), corresponding to the stage of kinematic chain without fault;

b) increase of signal amplitude over the value of $800 \mathrm{~mA}$, accompanied by an increase of motor housing temperature over $60^{\circ} \mathrm{C}$, corresponding to the stage of worn out element in the kinematic chain( damaged bearing).

The parameters of electric motors (rated current and power consumption) and the increased temperature of motor housing can be indicators that signal the wear of an element of the kinematic chain during motor operation. In the results of our research, the increase of the value of current is associated with the beginning of an abnormal operation caused by the wear of some components that can be of critical nature.

This size can be controlled; therefore when a maximum value is reached, the decisionmaker (specialist of maintenance) can stop the system for adjustment/change of components (Figure 5).

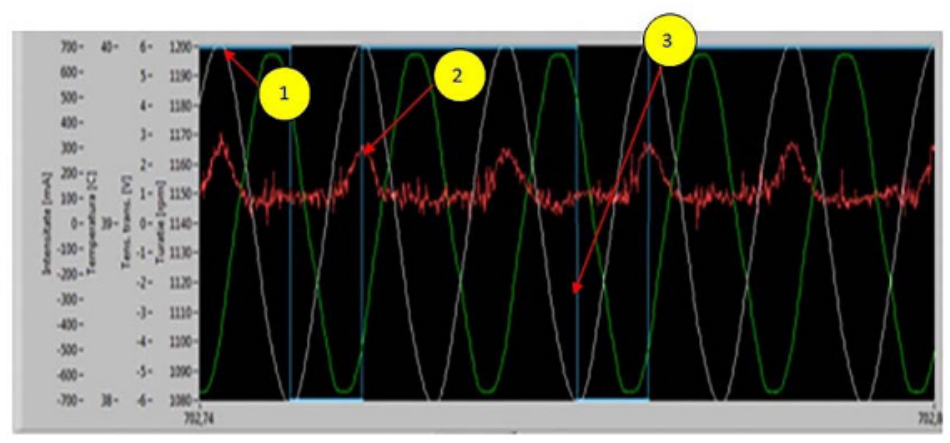

Fig.5. Virtual instrument of monitoring (current intensity- 1, temperature- 2, motor speed- 3), LabVIEW application.

As the operating hours number increases, the shape of current changes its amplitude. This size can be controlled and if there is a maximum value, the system can be stopped by the decision-maker (specialist of maintenance) for adjusting/replacing a component.

Table 1 shows the values of current, temperature and rotational speed. The increased values of current and temperature are obvious, also the decrease of the rotational speed entailed by the equipment running duration. We notice that during a normal operation the values are constant and the equipment operation keeps within the set scope - of normal operation.

By monitoring the system, we observed and recorded increased values of markers associated with the increase of the number of operation hours.

The research paper highlights the advantage of monitoring the parameters by means of markers set for the vital components of the equipment and helps the specialist to decide when to take action. 
Table 1 Measured values (normal $\square$, fault $\square$ ).

\begin{tabular}{|c|c|c|c|c|c|c|c|c|}
\hline No & $\begin{array}{c}\text { Time } \\
(\mathrm{h})\end{array}$ & $\begin{array}{c}\text { Amperage } \\
(\mathrm{A})\end{array}$ & $\begin{array}{c}\text { Speed } \\
(\mathrm{RPM})\end{array}$ & No & $\begin{array}{c}\text { Time } \\
(\mathrm{h})\end{array}$ & $\begin{array}{c}\text { Amperage } \\
(\mathrm{A})\end{array}$ & $\begin{array}{c}\text { Speed } \\
(\mathrm{RPM})\end{array}$ & $\begin{array}{c}\text { Temp } \\
\mathrm{C}^{\circ}\end{array}$ \\
\hline 1 & 1 & 0.125 & 1440 & 1000 & 10 & 0.7 & 1200 & 41 \\
\hline 2 & $1 \mathrm{~h} 30^{\prime}$ & 0.125 & 1440 & 1001 & $10 \mathrm{~h} 30^{\prime}$ & 0.7 & 1380 & 41 \\
\hline 3 & 2 & 0.125 & 1440 & 1002 & 11 & 0.71 & 1380 & 43 \\
\hline 4 & $2 \mathrm{~h} 30^{\prime}$ & 0.125 & 1440 & 1003 & $11 \mathrm{~h} 30^{\prime}$ & 0.72 & 1380 & 43 \\
\hline 5 & 3 & 0.125 & 1440 & 1004 & 12 & 0.72 & 1380 & 43 \\
\hline 6 & $2 \mathrm{~h} 30^{\prime}$ & 0.125 & 1440 & 1006 & $12 \mathrm{~h} 30^{\prime}$ & 0.72 & 1380 & 43 \\
\hline 7 & 4 & 0.125 & 1440 & 1007 & 14 & 0.73 & 1400 & 44 \\
\hline.. & $\ldots \ldots \ldots .$. & $\cdots \cdots \cdots \cdots$ & $\cdots \cdots \cdots$ & $\mathrm{n}$ & $\mathrm{n}+1$ & 0.85 & 1400 & 60 \\
\hline
\end{tabular}

Figure 6 shows the analysis of monitored markers values. This analysis helps the maintenance department to:

- make maintenance decisions at the most appropriate time;

- achieve a strategy of maintenance and to develop the responsibility of the involved staff;

- preserve the technical system reliability according to the recommendations of its manufacturer.

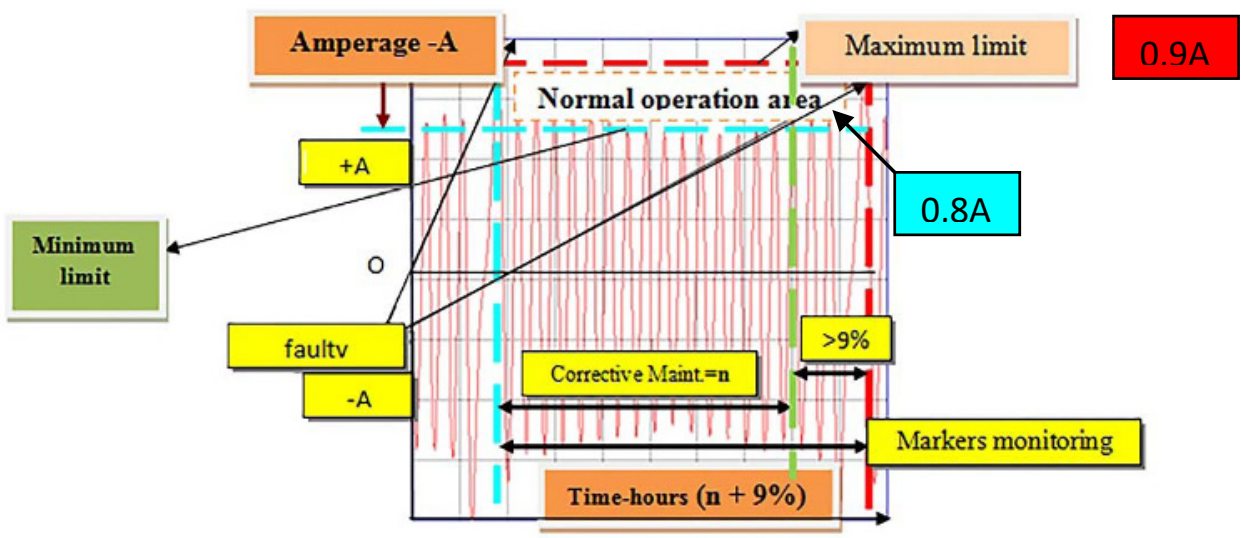

Fig.6. Monitoring of the operation area by electricity footprint.

\section{Conclusions}

The proposed research method allows obtaining valuable information that lead to the diagnosis of equipment/kinematic chains by using the monitoring and modern analysis methods. The thorough knowledge of equipment components enables to find the mechanisms that explain the response of the material to the action, generally destructive, of some exterior 
factors such as the mechanical, chemical or thermal ones. Also, the establishment and analysis of some suitable markers of maintenance can technically justify certain decisions. The selection of the most appropriate parameters to be monitored and the improvement of the analysis algorithms were the targets of the research whose results were presented in this paper. Regarding the scientific perspectives that this paper presents: a dynamic mathematical model can be determined in the future that can simulate the functioning of the equipment till the maintenance intervention.

\section{References}

[1] C. Bîrleanu, Student Scientific Session (May, 2015)

[2] http://en-us.fluke.com/site/fluke-sitemap/

[3] I. Păunescu, P. Păunescu, St. Velicu, Fourth International Conference MIDIS 2015Sibiu (2016)

[4] St. Velicu, I. Păunescu, D. M. Paraschiv, ICMAS 9, 1 (2014).

[5] Al. Velicu. Research on integrated maintenance of technological systems (UPB, 2010) 